\title{
Compressibility of air in fibrous materials
}

\author{
Tarnow, Viggo
}

\section{Published in:}

Acoustical Society of America. Journal

Link to article, DOI:

$10.1121 / 1.414790$

Publication date:

1996

\section{Document Version}

Publisher's PDF, also known as Version of record

Link back to DTU Orbit

Citation (APA):

Tarnow, V. (1996). Compressibility of air in fibrous materials. Acoustical Society of America. Journal, 99(5), 3010-3017. https://doi.org/10.1121/1.414790

\section{General rights}

Copyright and moral rights for the publications made accessible in the public portal are retained by the authors and/or other copyright owners and it is a condition of accessing publications that users recognise and abide by the legal requirements associated with these rights.

- Users may download and print one copy of any publication from the public portal for the purpose of private study or research.

- You may not further distribute the material or use it for any profit-making activity or commercial gain

- You may freely distribute the URL identifying the publication in the public portal

If you believe that this document breaches copyright please contact us providing details, and we will remove access to the work immediately and investigate your claim 


\section{Compressibility of air in fibrous materials}

Viggo Tarnow

Department of Applied Engineering Design and Production, Technical University of Denmark, Bygning 358, DK 2800 Lyngby, Denmark

(Received 10 April 1995; accepted for publication 8 December 1995)

The dynamic compressibility of air in fibrous materials has been computed for two assumed configurations of fibers which are close to the geometry of real fiber materials. Models with parallel cylinders placed in a regular square lattice and placed randomly are treated. For these models the compressibility is computed approximately from the diameter and mean distances between cylinders. This requires calculation of the air temperature, which is calculated for cylinders in a regular lattice by the Wigner-Seitz cell approximation. In the case of random placement, the calculation is done by a summation over thermal waves from all fibers, and by a self-consistent procedure. Figures of the compressibility in the frequency range $10-100000 \mathrm{~Hz}$, are given for diameter of the cylinders of $6.8 \mu \mathrm{m}$, and mean distances between them from 50 to $110 \mu \mathrm{m}$, which corresponds to glass wool with a density of 40 to $16 \mathrm{~kg} / \mathrm{m}^{3}$. When the theoretical values for random placement were compared with measurements, it turned out that the random model could not describe the experimental data. However, they could be described accurately by assuming that the fibers have a tendency to form pairs. (C) 1996 Acoustical Society of America.

PACS numbers: 43.58.Bh, 43.20.Hq, 43.20.Jr, 43.55.Ev

\section{LIST OF SYMBOLS}

A

$B$

$a$

$a_{0}$

$b$

$C(\omega)$

$C_{0}$

$c_{f}$

$c_{p}$

$D$

$d v$

$\exp (-i \omega t)$

F

$f$

$H_{0}^{1}(z), H_{1}^{1}(z)$

$H(z)$

$J_{0}(z), J_{1}(z)$

$J(z)$

K

$k$

$k_{1}$

$L$

$N$

n

mass area per cylinder

constant defined in (13)

radius of fiber or cylinder

proportional to monopole-strength of heat

source on cylinder

square root of area per fiber or mean dis-

tance between plates

dynamic compressibility of air

adiabatic compressibility

heat capacity of fibers per mass

heat capacity of air at constant pressure per

integration constant (27)

element of volume

complex time factor

characteristic frequency for plates

frequency

Hankel function of zero and first order and first kind

sum of Bessel functions (12)

Bessel functions of zero and first order

sum of Bessel functions (11)

heat capacity per volume of medium

thermal wave vector for air

thermal wave vector for effective medium

length of ensemble of plates

number of cylinders

normal to side of unit cell
$P$

$P_{0}$

$p(\omega)$

$R$

$\mathbf{r}$

$\mathbf{r}_{i}$

$T$

$T_{0}$

$T(\omega, r)$

$t$

$V_{0}$

$v(\omega)$

$x$

$y$

$z$

$\gamma$

$\delta$

$\kappa$

$\rho$

$\rho_{0}$

$\rho_{f}$

$\rho_{w}$

$\omega$

$\left\langle a^{2}\right\rangle$

$\langle T(\omega)\rangle$

$\langle\theta(\omega)\rangle$ air pressure

static air pressure

acoustic pressure

radius of cell

vector from origin of coordinate system to point in air

vector from origin to axis of cylinder

absolute temperature

static absolute temperature

temperature increase

time

static volume of air in material

increase of volume of air in material

distance between plates

space coordinate

complex variable

heat capacity of air at constant pressure/ heat capacity of air at constant volume thermal boundary-layer thickness

coefficient of thermal conduction of air

mass density of air

static mass density of air

mass density of fibers

mass density of glass wool

cyclic frequency

mean square radius of fibers

mean temperature

mean normalized temperature

\section{INTRODUCTION}

The velocity and the attenuation of sound in fibrous materials depend on the effective mass and the compressibility

of the air. In the following, the compressibility will be computed from the average distance between fibers and their diameters.

We assume that the compression is linear and mono- 
chromatic, and define the complex compressibility from the equation

$$
C(\omega)=-\frac{v(\omega)}{V_{0} p(\omega)},
$$

where $\omega$ is the cyclic frequency and $v(\omega)$ is the time varying volume of air in the material, $V_{0}$ the static volume, and $p(\omega)$ is the acoustical pressure.

The established way of predicting the acoustical properties of fiber materials is by use of the theory of porous materials, which has been described by many authors. ${ }^{1-12}$ In this theory, one assumes that the material consists of a matrix with circular pores of small radius. The compressibility of the air in the pores can be computed if one knows the radius of the pores, but for fibrous material it is not obvious how one finds the radius of the pores from the diameters of the fibers and their mean distances. The usual way of computing the compressibility uses the air flow resistance of the actual material to find the radius of the pores, and from the radius the compressibility is computed. ${ }^{5,9}$ Predictions in this way will later be compared with predictions from one of the models of this paper.

It is the goal of the work reported here to calculate the dynamic compressibility of fibrous materials from the microscopic geometry of the material without introducing adjustable parameters that are used to fit predictions from models to experimental data. The purpose is to obtain a better physical understanding of the dynamic compressibility of fiber materials, in order to be able to predict it when the microscopic geometry is known. The diameters of the fibers can be found by microscopy and the volume density from mass density.

It is not possible to calculate the compressibility from the models of the theory of porous materials, because one cannot calculate the radius of the tubes of the theory from the diameters and volume density of fibers.

We consider models that have a geometry similar to the microscopic geometry of fiber materials. In the models it is assumed that the fibers all have the same diameter and are parallel. A model with fibers in a regular square lattice is first treated, and then follows a model with parallel fibers randomly placed.

The results of the calculation of compressibility was compared with measurements and it turned out that results of the calculations from the diameters of fibers and their density did not agree with the experimental data. In order to obtain agreement one had to assume a lower density of the fibers than calculated from the mass density.

This shows that in order to calculate the dynamic compressibility from first principles, one has to have more information of the microscopic geometry than the mean diameter of fibers and their volume density.

\section{GENERAL THEORY}

The compressibility of air between fibers depends on the temperature rise during compression, which is isothermal at low frequencies and adiabatic at high ones. By standard ther- modynamics one may show that the dynamic compressibility $C(\omega)$ divided by the adiabatic compressibility $C_{0}$ can be computed from

$$
\frac{C(\omega)}{C_{0}}=\gamma-(\gamma-1)\langle\theta(\omega)\rangle,
$$

where $\gamma=1.40$ is the heat capacity of air at constant pressure divided by the heat capacity at constant volume, and the normalized temperature increase is

$$
\langle\theta(\omega)\rangle=\frac{\rho_{0} c_{p}}{p(\omega)} \frac{1}{V_{0}} \int_{V} T(\omega) d v,
$$

where $\rho_{0}$ is the static density of air, $c_{p}$ is the compressibility per mass of air at constant pressure, $T(\omega)$ the temperature increase, and the integral is taken over the volume of air between the fibers $V_{0}$. The derivation of Eqs. (2) and (3) is given in Appendix B.

In order to compute the compressibility from (2) and (3), one has to know the temperature increase of the air for a given pressure increase. The temperature can be found from the following differential equation,

$$
\kappa \nabla^{2} T(\omega)+\rho_{0} c_{p} i \omega T(\omega)=i \omega p(\omega),
$$

where $\kappa$ is the coefficient of thermal conductivity of air. This equation was given as Eq. (4) of Ref. 8. We use the following complex time factor

$$
e^{-i \omega t}
$$

where $t$ is the time.

The differential equation for the temperature Eq. (4) shall be solved by using the proper boundary condition on the fibers. In the literature ${ }^{1-12}$ one assumes that the temperature rise on the fibers is zero. But this is not accurate for light commercial glass wool, because the heat capacity of the glass fibers per volume is not infinitely great compared to the heat capacity of the air. The proper boundary condition is that the heat flow from the air to the fiber is continuous on the surface of the fiber

$$
\left.2 \pi a \kappa \frac{\partial T(\omega)}{\partial r}\right|_{r=a}=c_{f} \rho_{f} \pi a^{2}(-i \omega) T(\omega),
$$

where $a$ is the radius of the cylinder (fiber), $r$ is the radius of a polar coordinate system with center in the cylinder, $c_{f}$ is the heat capacity per mass of the fiber glass, $\rho_{f}$ is the mass density of the fibers.

The left-hand side of (6) is the surface area of a unit length fiber times the heat flux vector for air, this equals the heat flow per time to the piece of fiber. The right-hand side is the heat capacity of the same piece of fiber times the temperature increase per time, this equals the increase per time of the heat contents in the piece of fiber.

\section{CYLINDERS IN REGULAR SQUARE LATTICE}

A reasonable model of fiber material consists of parallel cylinders because fibers in glass wool tend to be parallel. It is difficult to compute exactly the compressibility for this model, therefore one has to use approximate computations, but the problem is not too difficult to solve, because the 


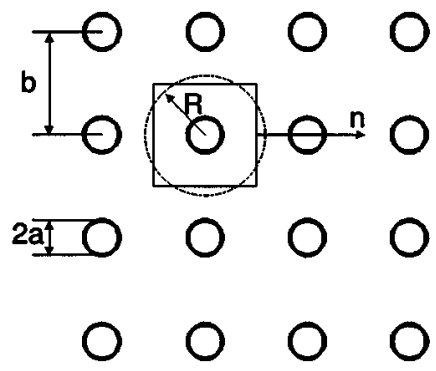

FIG. 1. Cylinders placed in regular square lattice.

concentration of fibers is small in glass fiber materials. The volume concentration of fibers is $1.6 \%$ in heavy glass wool with a mass density of $40 \mathrm{~kg} / \mathrm{m}^{3}$.

A model with parallel fibers in a regular square lattice is discussed in this section, and later a model with parallel fibers placed randomly is treated. The model consists of cylinders, with equal radius, parallel and placed in a regular lattice, Fig. 1. This is not a very realistic model of the actual fiber material, but it is discussed here because it is easier to calculate the temperature in this case than for a model with random placement of the cylinders, and the results from the two models will be compared.

We use a cylinder coordinate system with origin on the axis of one cylinder as shown in Fig. 1, and a square unit cell of the lattice. From the symmetry of the lattice it follows that the boundary condition on the sides of the unit cell is

$$
\frac{\partial T(\omega)}{\partial n}=0
$$

where $n$ is a normal to a side of the unit cell.

Equation (4) is to be solved with the boundary conditions (7) and (6), but the square boundary of the unit cell is replaced by a circle with the area of the unit cell, Fig. 1. The boundary condition is now

$$
\left(\frac{\partial T(\omega)}{\partial r}\right)_{r=R}=0 \text { with } R=\frac{b}{\sqrt{\pi}},
$$

where $R$ is the radius of the circle, and the square unit cell has side length $b$.

The computation method is similar to the Wigner-Seitz approximation, which was used to compute the cohesive energy of metals; it is described in Sec. 10-13 of Ref. 13, which contains references to the original literature.

Equation (4) with the boundary conditions (6) and (8) can be solved by Bessel functions. The result for the compressibility is

$$
\begin{aligned}
\frac{C(\omega)}{C_{0}}= & \gamma-(\gamma-1)\left[1+\frac{2 \pi k a}{\pi\left(R^{2}-a^{2}\right)}\right. \\
& \left.\times\left(\frac{H_{1}^{1}(k R) J_{1}(k a)-H_{1}^{1}(k a) J_{1}(k R)}{H_{1}^{1}(k R) J(k a)-H(k a) J_{1}(k R)}\right)\right],
\end{aligned}
$$

where $a$ is the radius of the cylinders, and $k$ the thermal wave vector, which can be written as $(1+i) / \delta$, where $\delta$ is called the thermal boundary-layer thickness,
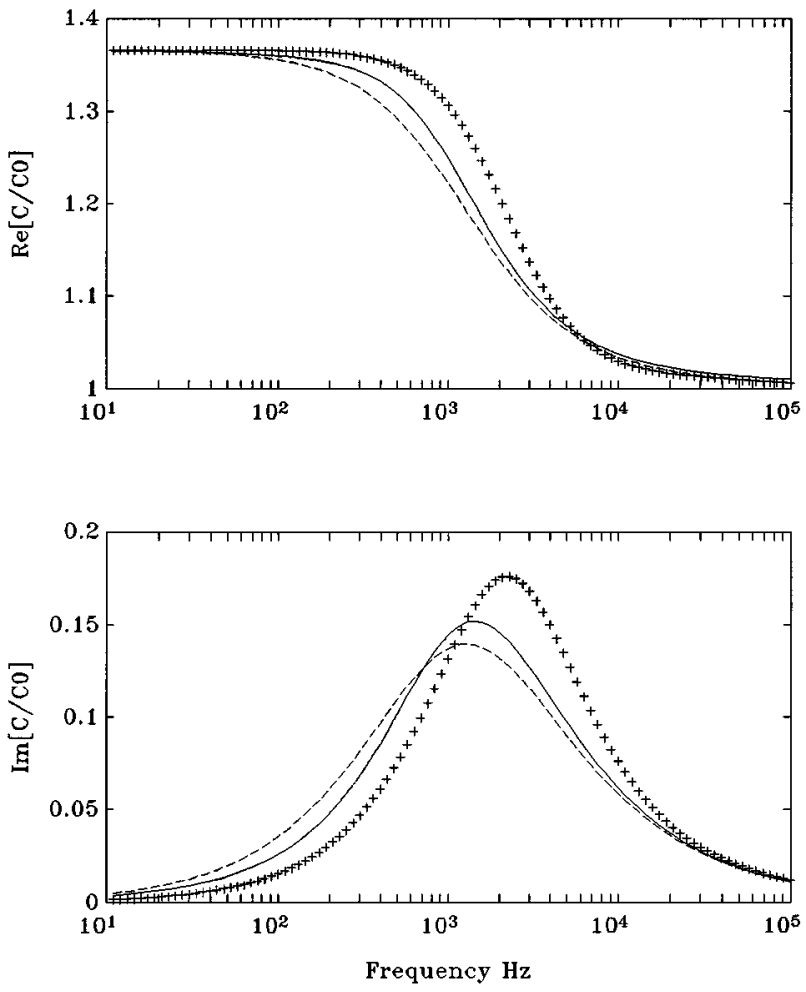

FIG. 2. The real and imaginary part of the dynamic compressibility divided by the adiabatic one. The compressibility was calculated for parallel cylinders placed in a regular lattice and randomly. The curves with crosses is for placement in a regular lattice. The full line is for random placement, calculated by the self-consistent method, and the broken line by the sum method. The radius of the cylinders is $3.4 \mu \mathrm{m}$, and the mean distance between cylinder axes is $75 \mu \mathrm{m}$. These figures are appropriate for glass wool with a density of $16 \mathrm{~kg} / \mathrm{m}^{3}$.

$$
k=\sqrt{\frac{i \omega \rho_{0} c_{p}}{\kappa}},
$$

and $H_{0}^{1}(z)$ is the Hankel function of the zero order and first kind of the complex variable $z, J_{0}(z)$ is the Bessel function of the zero order, and

$$
J(k a)=J_{0}(k a)-B J_{1}(k a)
$$

and

$$
H(k a)=H_{0}^{1}(k a)-B H_{1}^{1}(k a),
$$

where

$$
B=\frac{2 \kappa k}{i \omega a c_{f} \rho_{f}} .
$$

The result of a computation is shown in Fig. 2 as crosses, for $a=3.4 \mu \mathrm{m}$ and $b=75 \mu \mathrm{m}$, which corresponds to a density of glass wool of $16 \mathrm{~kg} / \mathrm{m}^{3}$.

\section{CYLINDERS PLACED RANDOMLY: TEMPERATURE BY SUMMATION}

We now assume that the cylinders are randomly distributed, but still parallel. The method of the computation is similar to the one used by Rayleigh, ${ }^{14}$ who computed the conductivity of a medium with cylinders in a rectangular 


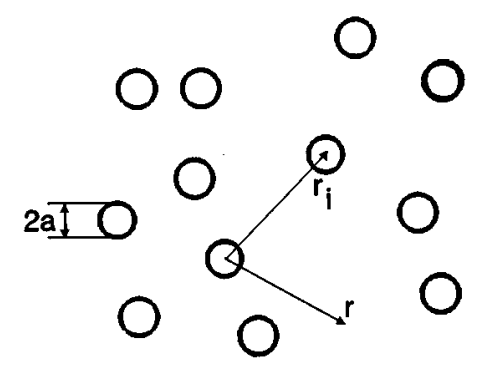

FIG. 3. Random placement of cylinders.

lattice. The temperature rise can be written as the temperature rise without cylinders plus a temperature wave from each cylinder

$$
T(\omega, r)=\frac{p(\omega)}{\rho_{0} c_{p}}+\sum_{i} a_{0} H_{0}^{1}\left(k\left|\mathbf{r}-\mathbf{r}_{i}\right|\right),
$$

where $a_{0}$ is proportional to the monopole strength of heat sources on the cylinders, and $\mathbf{r}$ is a vector from the origin of the coordinate system to a point in the air, and $\mathbf{r}_{i}$ is a vector from the origin to the center of a cylinder, Fig. 3. In order to fulfill the boundary condition, one places a cylinder at the origin, and sets Eq. (14) into the boundary condition (6), and puts the field point $\mathbf{r}$ in (14) on the surface of the cylinder at the origin. Thus

$$
\frac{p(\omega)}{\rho_{0} c_{p}}+a_{0}\left[H_{0}^{1}(k a)+\sum_{i}{ }^{\prime} H_{0}^{1}\left(k r_{i}\right)-B H_{1}^{1}(k a)\right]=0,
$$

where the summation now excludes the cylinder at the origin. The sum can be replaced by an integral because the cylinders are randomly distributed, and we want a mean value of $a_{0}$. The cylinders are assumed to be distributed with constant density in the plane, therefore

$$
\sum^{\prime} H_{0}^{1}\left(k r_{i}\right)=\frac{N}{A} \int_{2 a}^{\infty} H_{0}^{1}(k r) 2 \pi r d r,
$$

$N$ is the number of cylinders in area A. After integration, the result is

$$
a_{0}=\frac{-p(\omega) /\left(\rho_{0} c_{p}\right)}{H_{0}^{1}(k a)-B H_{1}^{1}(k a)-N 4 \pi a H_{1}^{1}(2 k a) /(A k)},
$$

The mean temperature can be found from (14)

$$
\langle T(\omega)\rangle=\frac{p(\omega)}{\rho_{0} c_{p}}+\frac{a_{0}}{A} \int_{S} \sum^{\prime} H_{0}^{1}\left(k\left|\mathbf{r}-\mathbf{r}_{i}\right|\right) d S,
$$

where one integrates over a plane perpendicular to the axes of the cylinders. From (18) one gets the normalized temperature rise, defined in (3),

$$
\langle\theta(\omega)\rangle=1+\frac{1}{A} \frac{\rho_{0} c_{p}}{p(\omega)} a_{0} \int_{s} \sum^{\prime} H_{0}^{1}\left(k\left|\mathbf{r}-\mathbf{r}_{i}\right|\right) d S .
$$

Exchange the integration and summation. Each integral is equal, as can be seen by changing the origin of the coordinate system for the $i$ th term to $r_{i}$, and remember that the integration is over the whole plane. Therefore

$$
\langle\theta(\omega)\rangle=1+\frac{N}{A} \frac{\rho_{0} c_{p}}{p(\omega)} a_{0} \int_{a}^{\infty} H_{0}^{1}(k r) 2 \pi r d r .
$$

Integration yields

$$
\langle\theta(\omega)\rangle=1-a_{0} \frac{N \rho_{0} c_{p} 2 \pi a H_{1}^{1}(k a)}{A p(\omega) k} .
$$

If one sets Eq. (17) into (21), one obtains the normalized temperature increase. The dynamic compressibility divided by the adiabatic one is found from (2), (17), and (21). The broken line in Fig. 2 shows the calculated compressibility ratio as a function of frequency for $a=3.4 \mu \mathrm{m}$ and $b=75$ $\mu \mathrm{m}$, where the square root of the area per fiber $b$, is defined by

$$
b^{2}=\frac{A}{N} .
$$

A simple formula for the compressibility can be useful. It can be found by assuming infinite heat capacity of cylinders, and expanding the Bessel functions in series. In this way, one obtains

$$
\frac{C(\omega)}{C_{0}}=\gamma-\frac{\gamma-1}{1+4 i /(k b)^{2}[(2 i / \pi) \ln (0.89054 k a)+1]},
$$

which is a good approximation for $a<6 \mu \mathrm{m}$.

The computation can be checked in the high-frequency limit by a simple physical argument, because the thermal boundary layer is thin at high frequencies. When it is thinner than the mean distance between cylinders, one need only think of one cylinder. Therefore only one term in Eq. (14) needs to be kept, and the temperature at the cylinder in (14) is zero at high frequencies; thus

$$
a_{0}=-\frac{p(\omega) / \rho_{0} c_{p}}{H_{0}^{1}(k a)} .
$$

This yields

$$
\frac{C(\omega)}{C_{0}}=1-(\gamma-1) \frac{2 \pi a H_{1}^{1}(k a)}{\left(b^{2}-\pi a^{2}\right) k H_{0}^{1}(k a)} .
$$

It can be shown by expanding in series that the highfrequency limit of the compressibility ratio computed from (21) is equal to (25).

The low-frequency limit of the compressibility is much more difficult to compute from simple physical arguments, because the heat waves from each cylinder have a very long range at low frequencies, and multiple reflections from the cylinders must be taken into account. Therefore the computation is a little uncertain at low frequencies, but this will be improved by the calculation in the following section.

\section{SELF-CONSISTENT CALCULATION}

The foregoing calculation of the temperature by summation of contributions of thermal waves from all cylinders to the one at the origin of the coordinate system, neglected reflections of thermal waves from the cylinders between the start point of the wave and the origin. This is not accurate at low frequencies, where the thermal boundary layer contains 
many cylinders. It is very difficult to take into account all these reflections directly, therefore a self-consistent method of computation is used, which is thought to be new in this connection. Similar methods have been used in the theory of flow resistance of fibers. ${ }^{15}$

The heat equation for air is modified by adding the unknown mean heat capacity per volume of the fibers, $K$. This equation is solved, and one finds the heat flow per time to the cylinder at the origin. The self-consistent condition is that this heat flow is set equal to the heat flow to $K$ times the volume per fiber. From this one finds $K$ and from $K$ the temperature.

Instead of Eq. (4), we solve a new equation

$$
\kappa \nabla^{2} T(\omega)+i \omega\left(\rho_{0} c_{p}+K\right) T(\omega)=i \omega p(\omega),
$$

where $K$ is a constant equivalent to the effective heat capacity per volume of the medium. This equation is solved with one cylinder at the origin of the coordinate system and the boundary condition in Eq. (6) for this cylinder.

A solution to Eq. (26) is

$$
T(\omega)=T_{1}-D H_{0}^{1}\left(k_{1} r\right),
$$

where

$$
T_{1}=\frac{p(\omega)}{\rho_{0} c_{p}+K},
$$

$T_{1}$ and $D$ are constants, and the wave vector is

$$
k_{1}=\sqrt{\frac{i \omega\left(\rho_{0} c_{p}+K\right)}{\kappa}} .
$$

When the boundary condition Eq. (6) is used, one gets

$$
D=\frac{T_{1}}{H_{0}^{1}\left(k_{1} a\right)-2 \kappa k_{1} H_{1}^{1}\left(k_{1} a\right) / i \omega c_{f} \rho_{f} a} .
$$

The heat energy that flows into one cylinder divided by the mean area per cylinder, equals the heat energy that flows to the equivalent heat capacity per area. Thus

$$
-K i \omega T_{1}=\frac{2 \pi a \kappa D k_{1} H_{1}^{1}\left(k_{1} a\right)}{b^{2}},
$$

where $b^{2}$ is the mean area per cylinder. In order to find $K$ we set (30) into (31), and find

$$
K=-\frac{2 \pi a \kappa k_{1} H_{1}^{1}\left(k_{1} a\right) /\left(i \omega b^{2}\right)}{H_{0}^{1}\left(k_{1} a\right)-2 \kappa k_{1} H_{1}^{1}\left(k_{1} a\right) / i \omega c_{f} \rho_{f} a} .
$$

Equations (29) and (32) are transcendental equations from which $k_{1}$ can be computed by iteration. First one assumes that $K$ is zero, and calculates $k_{1}$ from (29) then $K$ is found from (32). A new value of $k_{1}$ is found from (29), and this procedure is continued until $K$ has a stable value. The calculations for the graphs were done with 4 iterations, because the graphs were unchanged after the 4th iteration. The mean temperature rise can be found by

$$
T(\omega)=\frac{p(\omega)}{\rho_{0} c_{p}+K} .
$$
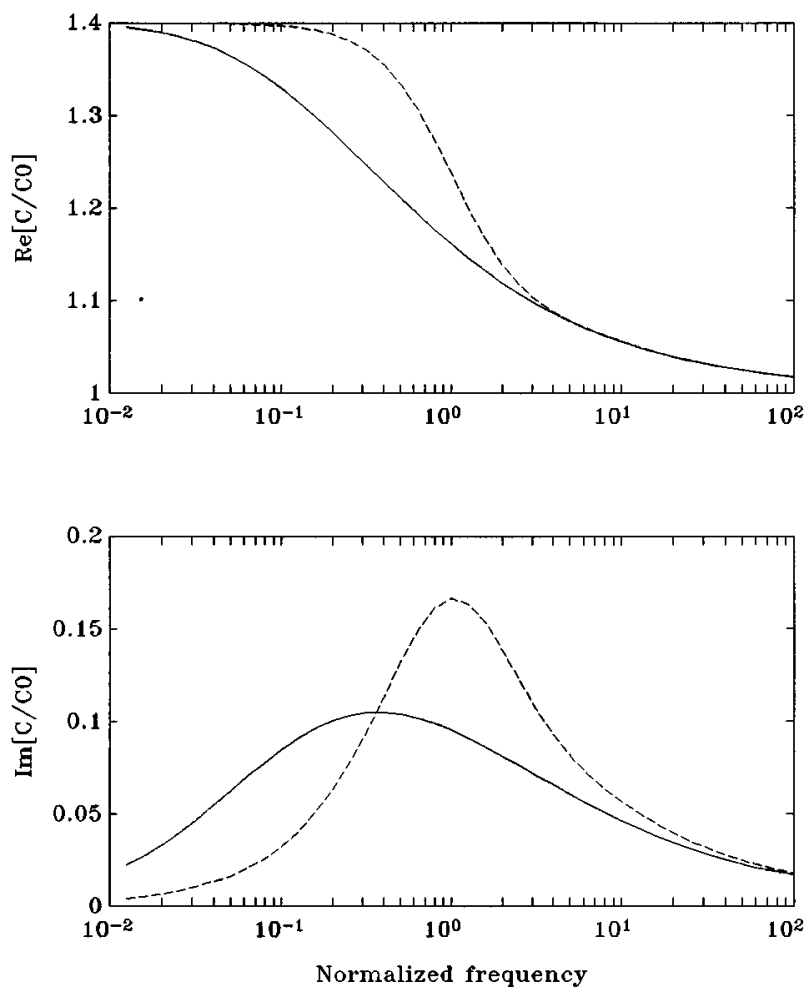

FIG. 4. The dynamic compressibility divided by the adiabatic one calculated for parallel plates. The unit of the horizontal axis is $F$ in Eq. (A3). The top of the figure shows the real part and the bottom the imaginary part. The broken line is for plates with equal distance, and the solid line is for randomly placed plates.

From this formula and (3) the normalized temperature rise is found, and the compressibility can be computed by (2). The result is shown as the full line in Fig. 2.

\section{DISCUSSION OF CALCULATIONS}

When the cylinders are placed in a periodic lattice, the change from isothermal to adiabatic compression occurs in a narrower frequency band than in the case of random placement. The transition from isothermal to adiabatic compression happens at a frequency where the thermal wavelength is about the same length as the distance between neighbor cylinders. The density of fibers measured from one fiber is discontinuous for a regular lattice, Figs. 1-2 of Ref. 13. This means that the distance to the nearest neighbor cylinders is a single number. The corresponding density in the case of random placement is continuous, and the distances to the nearest neighbors are distributed over a range in this case. Therefore the transition is more gradual in the case of random placement of cylinders. This is confirmed by a computation of compressibility for a one-dimensional slab geometry, because in this case one can make an exact computation. The results are shown in Fig. 4, and the details of the calculation are in the Appendix. It seems that the most reasonable model of fiber materials is the one with random placement of cylinders.

The computation in which one sums over waves from the cylinders is not reliable at low frequencies because multiple scattering of the thermal waves has not been taken into account. 
The self-consistent computation is thought to be the better. This is confirmed by the fact that the self-consistent method gives a precise value for the resistance to direct current air flow parallel to parallel fibers with random placement according to table VII of Ref. 16. The differential equation for the velocity in this case is the same as for the temperature; and the boundary condition the same, if one assumes infinite heat capacity of the cylinders. It is therefore reasonable to assume that the self-consistent method is reliable in the present case.

The zero frequency compressibility divided by the adiabatic compressibility is different from 1.40 (for air), because the heat capacity of the fibers is finite. The zero frequency compressibility divided by the adiabatic one can by calorimetric calculations be shown to be

$$
\frac{C(0)}{C_{0}}=\frac{1+\gamma\left(c_{f} \rho_{w} / c_{p} \rho_{0}\right)}{1+c_{f} \rho_{w} / c_{p} \rho_{0}} .
$$

\section{COMPARISON WITH MEASUREMENTS}

The compressibility has been measured for glass wool manufactured by Glasuld, Scan-Gobain Glass, Denmark. The mean diameter of the fibers was $6.8 \mu \mathrm{m}$, and their standard deviation was $2.7 \mu \mathrm{m}$. The mean distance between fibers can be found from the mass density of the glass wool $\rho_{w}$ and the mass density of the fibers $\rho_{g}$,

$$
b=\sqrt{\pi\left\langle a^{2}\right\rangle \rho_{g} / \rho_{w}},
$$

where $\left\langle a^{2}\right\rangle$ is the mean value of the square of the radii. For glass wool with a density of $16 \mathrm{~kg} / \mathrm{m}^{3}$, the mean distance between fibers was $b=75 \pm 5 \mu \mathrm{m}$. The compressibility was measured in the frequency range $30-1600 \mathrm{~Hz}$. The measurement method is described in Ref. 17.

The measurements were compared with the selfconsistent computation with $a=3.4 \mu \mathrm{m}$ and $b=75 \mu \mathrm{m}$, and this is shown as with crosses in Fig. 5. The fit between the measured and computed values is not perfect. The reason for the discrepancy between the theoretical curve and the experimental one could be explained by assuming that the fibers tend to assemble in pairs. If this is the case, the effective distance between the fibers becomes greater than the value found from the mass density and the assumption that each fiber is place randomly. This view is confirmed by the fact that a good fit can be obtained with $a=5 \mu \mathrm{m}$ and $b=112$ $\mu \mathrm{m}$, where $b$ was found from Eq. (35). If we replace the pair with one fiber with the same area as the pair, the new radius becomes $a=3.4 \cdot \sqrt{2}=4.8 \approx 5 \mu \mathrm{m}$. The density of the glass wool is the same, therefore according to (35), $b=75 \cdot \sqrt{2}$ $=106 \approx 112 \mu \mathrm{m}$. This is shown as the solid line in Fig. 5 . The prediction of the Allard/Champoux empirical model from Eq. (6) of Ref. 9 is shown as the broken line, which was based on a resistance of $5500 \mathrm{~kg} \mathrm{~s}^{-1} \mathrm{~m}^{-3}$, which was measured by a resonance method described in Sec. II of Ref. 17.

Measurements have also been done on glass wool with a density of $40 \mathrm{~kg} / \mathrm{m}^{3}$, and the results are shown in Fig. 6 . In this case the fibers seem to form pairs to a higher degree than in the low density case, because a good fit between the calculation and the measurements requires a radius of $7 \mu \mathrm{m}$.
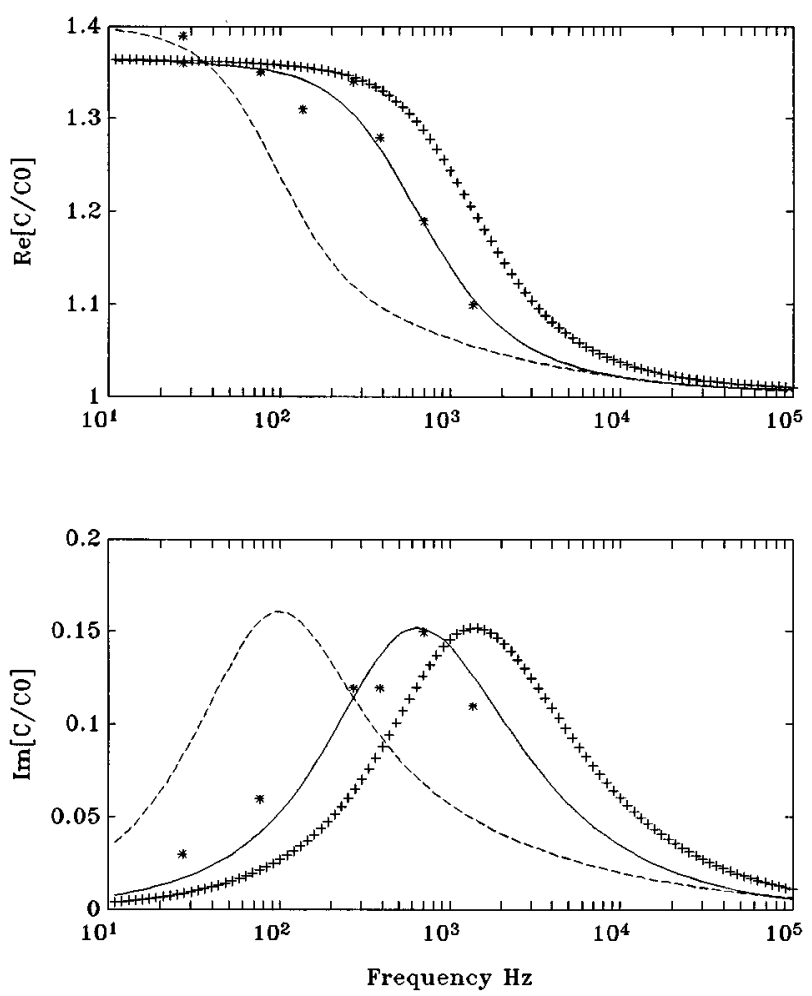

FIG. 5. Experimental values compared with theoretical ones for glass wool with a density of $16 \mathrm{~kg} / \mathrm{m}^{3}$. The cross curve was computed by the selfconsistent method, with $a=3.4 \mu \mathrm{m}$ and $b=75 \mu \mathrm{m}$, and the full line in the same way but with $a=5 \mu \mathrm{m}$ and $b=112 \mu \mathrm{m}$. The broken line shows the results of the Allard/Champoux empirical model, and was computed from the flow resistance of $5500 \mathrm{~kg} \mathrm{~s}^{-1} \mathrm{~m}^{-3}$.

The broken line in the figure is calculated by the theory of porous materials Eq. (6) of Ref. 9, with a resistance of 16900 $\mathrm{kg} \mathrm{s}^{-1} \mathrm{~m}^{-3}$, which was found as the value that gave the best fit to data for sound wave vector, and impedance of Figs. 6 and 8 of Ref. 17.

The heat transport from air to fibers is determined by the microscopic geometry of the glass wool. The fibers seem to be almost parallel when viewed in a microscope, it is therefore reasonable to assume parallel fibers.

In the theory it was assumed that the fibers all have the same diameter, which is not the case for real material, but this has only a limited influence on the computation of the compressibility, because the compressibility depends on the logarithm of the fiber radius as one can see from Eq. (23). But the computations are sensitive to the distance between fibers.

The experimental data for the real part of the compressibility seems to approach asymptotically a lower limit below 1.40 when the frequency approaches zero as would be expected from Eq. (34). From this equation one gets 1.36 for light glass wool with a mass density of $16 \mathrm{~kg} / \mathrm{m}^{3}$, this is close to the experimental value.

\section{CONCLUSION}

The compressibility of air in fiber material has been calculated for models of the fiber material consisting of parallel cylinders placed in a regular lattice and randomly. In the last case the calculations of temperature were done by summing 

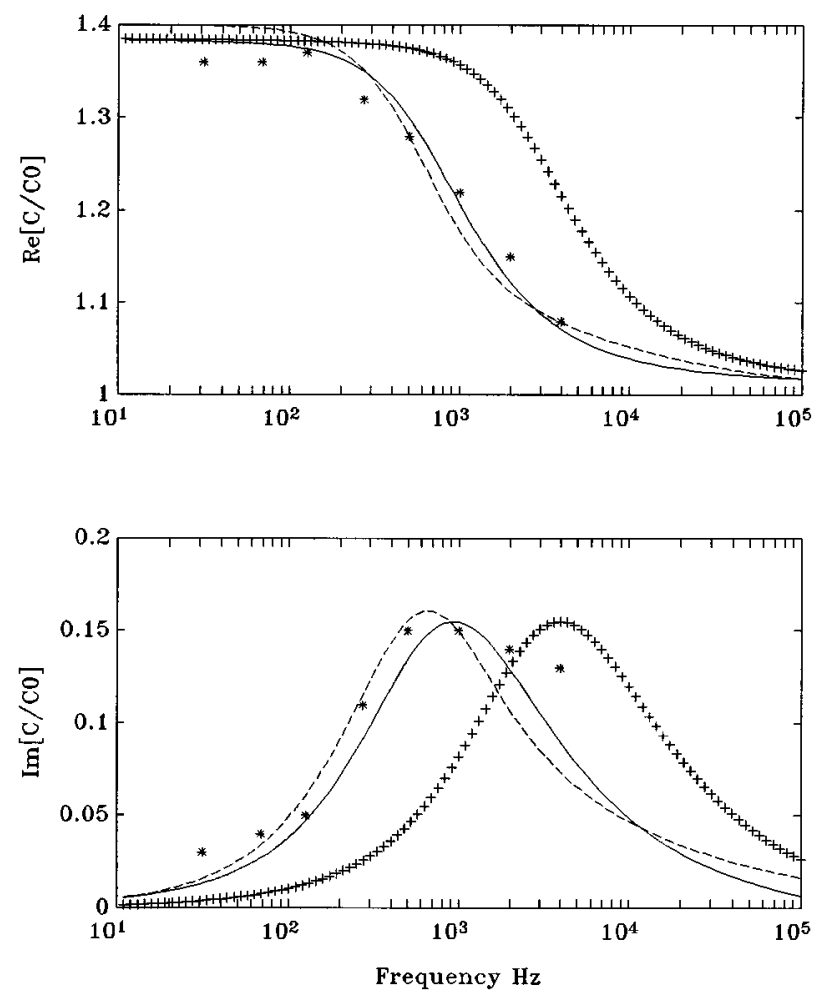

FIG. 6. Experimental values compared with theoretical ones for glass wool with a density of $40 \mathrm{~kg} / \mathrm{m}^{3}$. The cross curve was computed by the selfconsistent procedure, with $a=3.4 \mu \mathrm{m}$ and $b=48 \mu \mathrm{m}$, and the full line in the same way but with $a=7 \mu \mathrm{m}$ and $b=99 \mu \mathrm{m}$. The broken line shows the results of the Allard/Champoux empirical model, and was computed from a flow resistance of $16900 \mathrm{~kg} \mathrm{~s}^{-1} \mathrm{~m}^{-3}$.

contributions from all cylinders, and by a self-consistent procedure, which gave results close to each other, but the selfconsistent method is thought to be the most reliable one. The result of the calculations was compared with measurements on glass wool of density 16 and $40 \mathrm{~kg} / \mathrm{m}^{3}$. This requires knowledge of the fiber radius, which was found by microscopy, and the distance between fibers, which was found from the mass density of the material and the fiber radius. But a radius greater than the observed one is required in order to fit the calculation to the measurements; this can be understood if one assumes that the fibers tend to form pairs close together.

\section{APPENDIX A: ONE-DIMENSIONAL MODELS}

One-dimensional models for compressibility that can be solved exactly will be treated, they consist of parallel plates of infinitely small thickness and infinite heat capacity. Two cases will be treated here. In the first case the distances between the plates are equal, and in the second case the distances are randomly distributed.

\section{Equidistant plates}

We treat now the case of equidistant plates, this has been treated by many authors. ${ }^{3,7}$ Equation (4) is solved with $T(\omega)=0$ at the plates, and we find the mean value of the temperature increase. It is most easily found if one uses a coordinate system with origin midway between two plates and axis perpendicular to the plates. The distance between the plates is $b$. One gets the mean temperature rise

$$
\langle T(\omega)\rangle=\frac{p(\omega)}{\rho_{0} c_{p}}\left[1-\frac{\tan (k b / 2)}{k b / 2}\right] .
$$

The compressibility divided by the adiabatic compressibility can be found when this equation is set into Eq. (3), and (3) into (2);

$$
\frac{C(\omega)}{C_{L}}=\gamma-(\gamma-1)\left[1-\frac{\tan (k b / 2)}{k b / 2}\right] .
$$

The broken line in Fig. 4 shows the ratio between the two compressibilities as a function of frequency. The horizontal axis is the frequency divided by the normalized frequency $F$, which equals

$$
F=\frac{\pi \kappa}{2 \rho_{0} c_{p} b^{2}} .
$$

\section{Randomly placed plates}

The case of random placed plates has not been described before. The prescription for calculating the mean temperature is

$$
\langle T(\omega)\rangle=\frac{1}{V} \int_{V} T(\omega, y) d v,
$$

where the volume $V$ contains many "fibers." For one member of a statistical ensemble of plates, the mean value is

$$
\langle T(\omega)\rangle=\lim _{L \rightarrow \infty} \frac{1}{L} \int_{0}^{L} T(\omega, y) d y,
$$

where $L$ is the total length of the ensemble and $y$ is a space coordinate perpendicular to the plates. We assume that the plates are placed randomly on a line with a mean distance of $b$. The distances between the plates are Poisson distributed, ${ }^{18}$ and the probability $d p$ of finding a plate distance between $x$ and $x+d x$ is

$$
d p=\frac{1}{b} e^{-x / b} d x
$$

The Poisson distribution was chosen because it is the most simple; the only parameter it requires is the mean density.

The integral of the temperature over the space between two plates is computed. The mean of this integral is computed and the mean temperature is found by dividing by the average distance between plates. Thus the mean value of the temperature is

$$
\langle T(\omega)\rangle=\frac{1}{b^{2}} \int_{0}^{\infty} e^{-x / b}\left[\int_{0}^{x} T(\omega, y) d y\right] d x,
$$

where $x$ is the distance between two plates, $y$ is the distance from one plate to a point in the air, and $T(\omega, y)$ is found from Eq. (36). The mean temperature rise becomes

$$
\langle T(\omega)\rangle=\frac{p(\omega)}{\rho_{0} c_{p}}\left[1-\frac{2}{k b} \int_{0}^{\infty} \tan \left(\frac{k b x}{2}\right) e^{-x} d x\right] .
$$


When this is substituted into Eq. (3), one gets from (2) the dynamic compressibility divided by the adiabatic compressibility

$$
\frac{C(\omega)}{C_{0}}=\gamma-(\gamma-1)\left[1-\frac{2}{k b} \int_{0}^{\infty} e^{-x} \tan \left(\frac{k b x}{2}\right) d x\right] .
$$

This is shown in Fig. 4 as the solid line. It is obvious that the change from an isothermal compression to an adiabatic one is much more gradual than in the case of regularly spaced plates with the same mean density. At high frequencies the two curves approach each other asymptotically, because the change from isothermal to adiabatic compression occurs in a thin skin layer on the plates. As long as the skin layer (boundary layer) is thinner than the mean distances between the plates, there is no difference between a regular and a random placement of the plates. But when the skin layer is thicker than the mean distance between the plates, the two arrangements give a different frequency dependence of the compressibility. The change from isothermal to adiabatic compression occurs over a much wider frequency range if the plates are randomly distributed.

\section{APPENDIX B: CALCULATION OF COMPRESSIBILITY FROM MEAN TEMPERATURE} by

The microscopic compressibility $C_{m}(\omega)$ can be defined

$$
C_{m}(\omega)=\frac{1}{\rho_{0}} \frac{\partial \rho}{\partial P},
$$

where $\rho$ is the mass density of air, and $P$ the air pressure. The ideal gas law can be written

$$
P=\rho c_{p}\left(1-\gamma^{-1}\right) T,
$$

where $T$ is the absolute temperature. If this is logarithmically differentiated one gets

$$
C_{m}(\omega)=\frac{1}{\rho_{0}} \frac{\partial \rho}{\partial P}=\frac{1}{P_{0}}-\frac{1}{T_{0}} \frac{\partial T}{\partial P},
$$

where $\rho_{0}$ is the static mass density, $P_{0}$ is the static air pressure, and $T_{0}$ is the static absolute temperature. We find the absolute temperature from the ideal gas law and set this into the last equation, thus

$$
C_{m}(\omega)=\frac{1}{P_{0}}-\frac{\rho_{0} c_{p}\left(1-\gamma^{-1}\right)}{P_{0}} \frac{\partial T}{\partial P} .
$$

We need the average of the compressibility $C(\omega)$ over a volume $V_{0}$ that contains many fibers:

$$
C(\omega)=\frac{1}{V_{0}} \int_{V} C_{m}(\omega) d v .
$$

The adiabatic compressibility is

$$
C_{0}=\frac{1}{\gamma P_{0}} .
$$

From (B4), (B5), and (B6) one gets

$$
\frac{C(\omega)}{C_{0}}=\gamma-\rho_{0} c_{p}(\gamma-1) \frac{1}{V_{0}} \int_{V} \frac{\partial T}{\partial p} d v,
$$

which is the same as Eqs. (2) and (3).

${ }^{1}$ C. Zwikker and C. W. Kosten, Sound Absorbing Materials (Elsevier, Amsterdam, 1949).

${ }^{2}$ R. F. Lambert, "The acoustical structure of highly porous open-cell foam,' J. Acoust. Soc. Am. 72, 879-887 (1982).

${ }^{3} \mathrm{~K}$. Attenborough, "Acoustical characteristics of rigid fibrous absorbents," J. Acoust. Soc. Am. 73, 785-799 (1983).

${ }^{4}$ R. F. Lambert, "'Propagation of sound in highly porous open-cell elastic foams,'” J. Acoust. Soc. Am. 73, 1131-1138 (1983).

${ }^{5}$ R. F. Lambert and J. S. Tesar, "Acoustic structure and propagation in highly porous, layered, fibrous materials,'” J. Acoust. Soc. Am. 76, 12311237 (1984).

${ }^{6}$ H.-S. Roh, W. P. Arnott, and J. M. Sabatier, "'Measurement and calculation of acoustical propagation constants in array of small air-filled rectangular tubes," J. Acoust. Soc. Am. 89, 2617-2624 (1991).

${ }^{7}$ Michael R. Stinson, "The propagation of plane sound waves in narrow and wide circular tubes, and generalization to uniform tubes of arbitrary cross-sectional shape,', J. Acoust. Soc. Am. 89, 550-558 (1991).

${ }^{8}$ Michael R. Stinson and Yvan Champoux, "Propagation of sound and the assignment of shape factors in models of porous materials having simple pore geometries,'” J. Acoust. Soc. Am. 91, 685-695 (1992).

${ }^{9}$ Jean-F. Allard and Yvan Champoux, "New empirical equations for sound propagation in rigid frame fibrous materials,'” J. Acoust. Soc. Am. 91, 3346-3353 (1992).

${ }^{10}$ J. F. Allard, Propagation of Sound in Porous Media (Elsevier Applied Science, London, 1993)

${ }^{11}$ M. Henry, P. Lemarinier, J.-F. Allard, J. L. Bonardet, and A. Gedeon, "Evaluation of the characteristic dimensions for porous sound-absorbing materials,' J. Appl. Phys. 77, 17-20 (1995).

${ }^{12}$ P. Lemarinier, M. Henry, J.-F. Allard, J. L. Bonardet, and A. Gedeon, "Connection between the dynamic bulk modulus of air in a porous medium and the specific surface,' J. Acoust. Soc. Am. 97, 3478-3482 (1995).

${ }^{13}$ A. J. Dekker, Solid State Physics (Prentice Hall, New Jersey, 1962).

${ }^{14} \mathrm{~J}$. W. S. Rayleigh, On the influence of obstacles arranged in rectangular order upon the properties of a medium,' Philos. Mag. 34, 481-502 (1892).

15 J. D. Howells, "Drag due to the motion of a Newtonian fluid through a space random array of small fixed rigid objects,' J. Fluid. Mech. 64, 449-475 (1974).

${ }^{16}$ A. S. Sangani and C. Yao, "Transport processes in random arrays of cylinders. II. Viscous flow,'” Phys. Fluids 31, 2435-2444 (1988).

${ }^{17} \mathrm{~V}$. Tarnow, "Measurement of sound propagation in glass wool,' $\mathrm{J}$. Acoust. Soc. Am. 97, 2272-2281 (1995).

${ }^{18}$ J. M. Ziman, Models of Disorder (Cambridge U.P., Cambridge, MA, 1979), Eq. (2.6), p. 39. 